\title{
Turbulence level effects on conventional reflectometry using 2D full-wave simulations
}

\author{
J. Vicente, ${ }^{1, a)}$ F. da Silva, ${ }^{1}$ S. Heuraux ${ }^{2}$ G. D. Conway, ${ }^{3}$ C. Silva, ${ }^{1}$ and T. Ribeiro ${ }^{3}$ \\ ${ }_{1}^{1}$ Instituto de Plasmas e Fusão Nuclear, Instituto Superior Técnico, Universidade de Lisboa, \\ ${ }^{2}$ Institut Jean Lamour, UMR 7198, CNRS-University of Lorraine, F-54506 Vandoeuvre, France \\ ${ }^{3}$ Max-Planck-Institut für Plasmaphysik, 85748 Garching, Germany
}

published online 27 August 2018)

\begin{abstract}
Numerical simulations are critical in improving the capabilities of microwave diagnostics. In this work, the 2D finite-difference time-domain full-wave code REFMUL was applied to broadband turbulent plasmas using the conventional reflectometry setup. Simulations were performed with O-mode waves, fixed frequency probing, and I/Q detection. The plasma density, determining O-mode propagation, was modeled as the sum of a slab background plasma with a fluctuating component following a Kolmogorov-like amplitude $k$-spectrum. The density turbulence level $\delta n_{\mathrm{e}} / n_{\mathrm{e}}$ was scanned over several orders of magnitude for simulated plasma flows of constant plasma velocity in either the radial or the poloidal direction. Simulations show trends, such as spectral broadening of the complex $\mathrm{A}(t) \mathrm{e}^{\mathrm{i} \varphi(t)}$ signals and increasing fluctuations in $\mathrm{A}(t)$ and $\varphi(t)$ with increasing $\delta n_{\mathrm{e}} / n_{\mathrm{e}}$, that are similar for both plasma flow directions. These together with possibilities to reconstruct a poloidal wavenumber spectrum are discussed in view of extending the measuring capabilities. The onset of non-linear effects associated with phase runaway, as previously observed with other 1D and 2D codes, as well as radial Doppler effects is also observed and discussed. https://doi.org/10.1063/1.5038756
\end{abstract}

\section{INTRODUCTION}

Full-wave simulations have been instrumental in understanding reflectometry measurements and plasma-wave interactions, such as Bragg backscattering or forward scattering. ${ }^{1}$ In this work, the response of ordinary wave polarization $(\mathrm{O}-$ mode) reflectometry to analytically prescribed turbulent plasmas in simplified slab geometry is studied by performing $2 \mathrm{D}$ simulations in a conventional setup (i.e., perpendicular incidence) for both cases of poloidal and radial plasma flows. Fixed frequency probing is employed, and the reflectometry complex amplitude $\mathrm{A}(t) \mathrm{e}^{\mathrm{i} \varphi(t)}$, phase $\varphi(t)$, and amplitude $\mathrm{A}(t)$ signals are characterized while scanning the plasma turbulence level.

\section{BRAGG BACKSCATTERING}

The most efficient scattering process of the probing wave is caused by density fluctuations that meet the Bragg resonant rule. ${ }^{2-4}$ For conventional reflectometry, Bragg backscattering can occur when the local radial wavenumber of the injected wave $k(\boldsymbol{r})$ is resonant with the radial wavenumber of a given Fourier component of the density fluctuations, $k_{\mathrm{rad}}=2 k(\boldsymbol{r})$. The Bragg relation holds up to the limit wavenumber $k_{\mathrm{rad}}=2 k_{\mathrm{o}}$ set by the vacuum wavenumber of the probing wave $k_{\mathrm{o}}$, near the plasma entrance. Moreover, only

a) Author to whom correspondence should be addressed: jvicente@ipfn. tecnico.ulisboa.pt fluctuations with $k_{\text {rad }}>2 k_{\mathrm{A}}$ can satisfy the Bragg condition, where $k_{\mathrm{A}}$ is the Airy wavenumber, $k_{\mathrm{A}}=0.63\left(k_{\mathrm{o}}^{2} L_{\mathrm{n}}{ }^{-1}\right)^{1 / 3}$ with $L_{\mathrm{n}}$ being the density gradient scale length. While the poloidal characteristics of turbulence should not be responsible for any backscattering, in $2 \mathrm{D}$ it is impossible to neglect the radiation pattern of the emitter which contains poloidal wavenumbers and could thus resonate with poloidal fluctuations. In the case of O-mode probing and small coherent perturbations in a linear radial profile, a linear relation between the phase perturbations of the reflectometer signal $\delta \varphi$ and the density perturbation amplitude $\delta n$ has been shown, ${ }^{5,6}$

$$
\delta \phi=\sqrt{\pi} k_{o} \sqrt{\frac{L_{n}}{k_{f}}} \frac{\delta n}{n_{c}},
$$

where $n_{\mathrm{c}}$ is the cutoff density and $k_{f}$ is a given wavenumber component of the density fluctuation spectrum. However, additional non-linear contributions to phase variations may occur due to higher orders of Bragg backscattering. ${ }^{7}$

\section{TURBULENCE MODEL}

For O-mode propagation, the plasma cutoff for a given probing wavenumber $k_{\mathrm{o}}$ depends only on the plasma electron density $n_{\mathrm{e}}$. Thus, a numerical plasma is sufficiently modeled by the definition of the electron density. In this work, the total density $n_{\mathrm{e}}(\boldsymbol{r}, t)=n_{\mathrm{eo}}(\boldsymbol{r}, t)+\delta n_{\mathrm{e}}(\boldsymbol{r}, t)$ accounts for a linear radial electron density profile $n_{\mathrm{eo}}(\boldsymbol{r}, t)$ and a broadband density turbulence term $\delta n_{\mathrm{e}}(\boldsymbol{r}, t)$. While the background profile is simply set through a given density gradient, the turbulence is defined by a sum of modes with random phase,, 8 


$$
\delta n_{e}=\sum_{i=i_{m}}^{i_{M}} \sum_{j=j_{m}}^{j_{M}} A(i, j) \cos \left[k_{x}(i) x+k_{y}(j) y+\phi(i, j)\right] .
$$

The coefficients $A(i, j)$ allow establishing a Kolmogorov-like amplitude $k$-spectrum, which is in line with several experimental observations in the edge fusion plasmas, ${ }^{10}$ while $\varphi(i, j)$ is a random generated phase that sets a particular turbulence snapshot with the target spectral conditions. The 2D $k$-spectrum is defined by constant amplitude at low $k$ and up to a chosen wavenumber $k_{\text {knee }}$ followed by a power law decay $k^{-\alpha}$ along both the $x$ - and $y$-directions, corresponding to the radial and poloidal directions in a tokamak. The wavenumber knee is set to $k_{\text {knee }}=400 \mathrm{rad} / \mathrm{m}$ or $k_{\text {knee }}=4 \mathrm{~cm}^{-1}$ in usual (perhaps misleading) notation. The amplitude rolls off dropping to $1 \%$ of the flat top value at $k=15 \mathrm{~cm}^{-1}$, along with a spectral index $\alpha=3$. A realization of the turbulence model is shown in Fig. 1.

The root mean square value $\delta n_{e, R M S}$ of the density fluctuations generated with the above model can be easily modified and sets the turbulence level at the radial position $r_{c}$ of the cutoff density in the slab linear profile,

$$
\frac{\delta n_{e}}{n_{e}} \equiv \frac{\delta n_{e, R M S}}{n_{e}\left(r_{c}\right)} \times 100 \% .
$$

The total density, obtained by adding the linear profile and the turbulent density terms, results in a radial variation of the local turbulence level, which increases toward the periphery of the plasma as the background density decreases (see Fig. 2). This radial trend is also in general agreement with the experimental observations at the edge and scrape-off layer regions of tokamaks. ${ }^{11}$ In this work, two different electron density gradient lengths $L_{\mathrm{n}}$ have been used for background profiles, corresponding to shallow (long $L_{\mathrm{n}} \approx 0.16 \mathrm{~m}$ ) and steep (short $L_{\mathrm{n}} \approx 0.05 \mathrm{~m}$ ) density profiles, for which the minimum Bragg wavenumbers $\left(2 k_{\mathrm{A}}\right)$ are $1.8 \mathrm{~cm}^{-1}$ and $2.6 \mathrm{~cm}^{-1}$, respectively. Once a total density matrix was generated, simulations were carried out imposing a bulk plasma movement in either the poloidal or radial direction. In both cases, the Taylor "frozen turbulence" hypothesis ${ }^{12}$ is assumed, which considers that advection contribution by turbulent eddies is small and that therefore the advection past a fixed point can be taken to
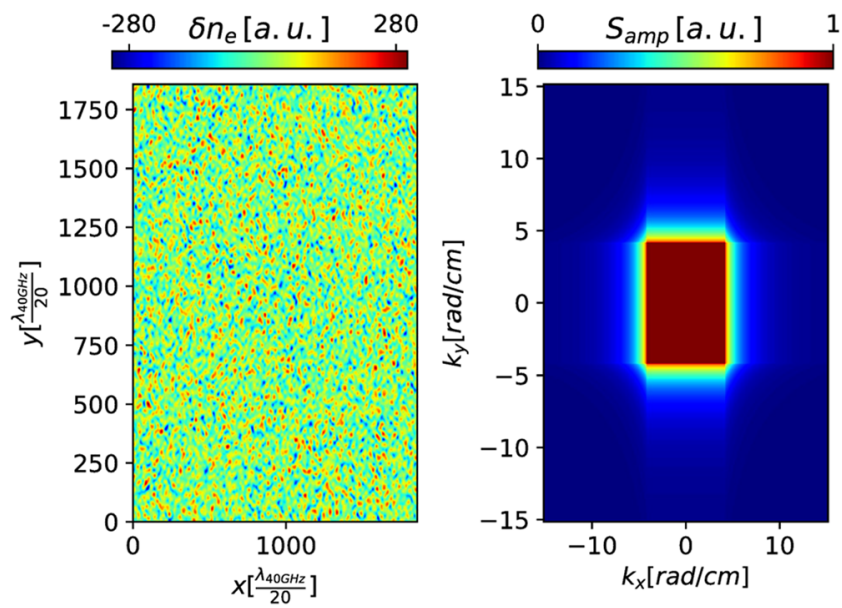

FIG. 1. Example of a 2D density turbulence realization (left) generated with a Kolmogorov-like model of a given amplitude $k$-spectrum (right).
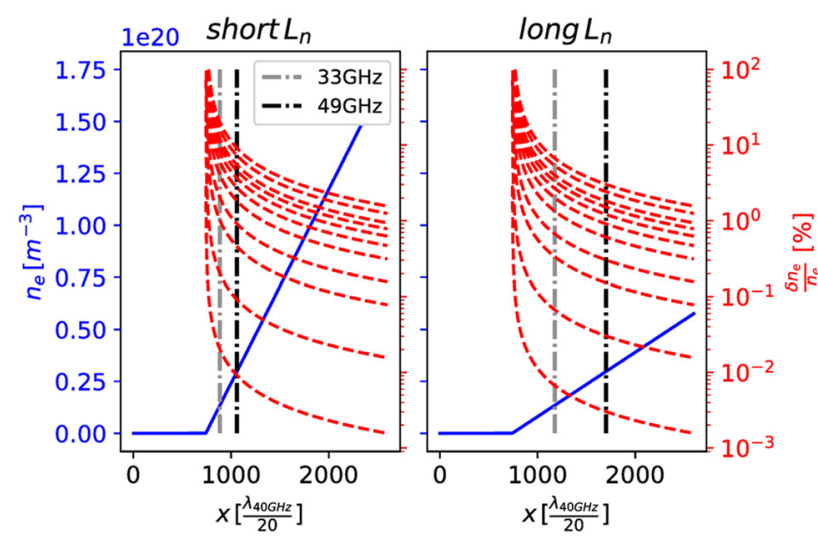

FIG. 2. Linear background density profiles (solid blue) and radial positions of the $f_{\mathrm{p}}=33 \mathrm{GHz}$ and $f_{\mathrm{p}}=49 \mathrm{GHz}$ cutoffs, together with the set of density turbulence level profiles (dashed red) employed for the numerical plasmas.

be entirely due to the mean flow. This hypothesis actually holds if the eddy mutation rate is much smaller than the mean plasma velocity.

\section{SIMULATION SETUP}

The full-wave code REFMUL used in this work solves the Maxwell equations for O-mode. ${ }^{13}$ Both electric and magnetic fields are coupled to the plasma electron density $n_{\mathrm{e}}(\boldsymbol{r}, t)$ which is used as a fusion plasma model, as mentioned in Sec. III. Simulations were designed with a monostatic setup, i.e., one antenna for both emission and reception. A unidirectional transparent source was used for injection of the signal allowing separation of the emitted probing wave from any returning waves. For directivity, a monostatic 2D H-plane horn antenna with a half-power beam width $\leq 6.5 \mathrm{~cm}$ at the plasma entry was employed. Probing waves with two different frequencies $f_{\mathrm{p}}=33 \mathrm{GHz}$ and $f_{\mathrm{p}}=49 \mathrm{GHz}$, corresponding to cutoff densities of $n_{c 33}=1.35 \times 10^{19} \mathrm{~m}^{-3}$ and $n_{c 49}=2.99 \times 10^{19} \mathrm{~m}^{-3}$, respectively, were used in separate runs. The two slab plasma profiles with the radial cutoff locations of the two probing frequencies that were used are shown in Fig. 2. Also shown are the several turbulence level profiles that were employed in both radial and poloidal plasma flow simulations.

The synthetic reflectometry phase $\varphi(t)$ and amplitude $\mathrm{A}(t)$ signals were obtained by performing identical simulation runs with sin and cos excitation signals allowing in-phase $\mathrm{I}(t)=\mathrm{A}(t) \cos (\varphi(t))$ and quadrature $\mathrm{Q}(t)=\mathrm{A}(t) \sin (\varphi(t))$ detection. These signals combined provide directly $\mathrm{A}(t)=\left[\mathrm{I}(t)^{2}\right.$ $\left.+\mathrm{Q}(t)^{2}\right]^{1 / 2}$ and $\varphi(t)=\mathrm{a} \tan [\mathrm{Q}(t) / \mathrm{I}(t)]$. The simulation box comprised a rectangular grid while the plasma itself was quadrangular with side length $\mathrm{L}=1859$ grid points since a radial block of the simulation box was used for the antenna setup and a vacuum distance $\left(\mathrm{d}_{\mathrm{vac}}=15.5 \mathrm{~cm}\right)$ to the plasma. The spatial grid resolution was $\Delta x=\Delta y=\lambda_{40 \mathrm{GHz}} / 20=3.75 \times 10^{-4} \mathrm{~m}$, where $\lambda_{40 \mathrm{GHz}}$ is the vacuum wavelength of an $f=40 \mathrm{GHz}$ wave frequency. The required time resolution of the simulations $\left(\Delta t=6.25 \times 10^{-13} \mathrm{~s}\right)$ impose that the duration of any simulated phenomena must be scaled down and any velocities involved scaled up. A rescaling is done such that $v_{\text {siml }}=R_{\mathrm{t}} v_{\text {real }}$, where $v_{\text {real }}$ is the characteristic velocity of a given plasma feature and $v_{\text {siml }}$ is the velocity in the simulation. To avoid relativistic 

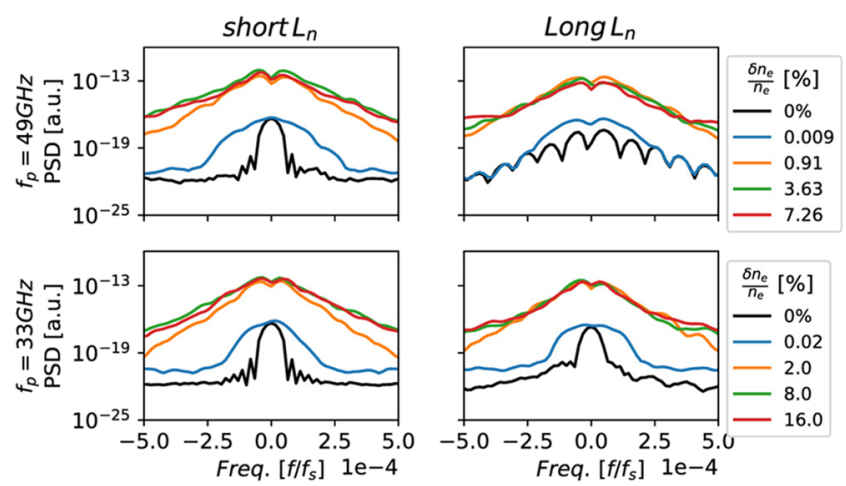

FIG. 3. Power spectra of complex amplitude signals $A(t) \mathrm{e}^{\mathrm{i} \varphi(t)}$ for turbulence level scans, in two different density gradient lengths $L_{\mathrm{n}}$ and two probing frequencies $f_{\mathrm{p}}$. Turbulence was generated using a Kolmogorov-like model and set to flow in the poloidal direction.

effects, the scaled-up velocities $v_{\text {siml }}$ are restricted to values of $v_{\text {siml }}<0.1 \mathrm{c}$, where $\mathrm{c}$ is the speed of light. In this work, simulations of plasma propagation in both radial and poloidal directions have been performed with $28 \times 10^{4}$ time iterations, corresponding to $v_{\text {siml }}=0.013 \mathrm{c}$.

\section{SIMULATION RESULTS}

\section{A. Poloidal plasma flow}

Independent simulations were performed for each turbulence level, in a range of $0.02 \%-20 \%$. The power spectra of the reflectometry complex amplitude signal $A(t) \mathrm{e}^{\mathrm{i} \varphi(t)}$ for the unperturbed profile case and a set of turbulence levels are shown in Fig. 3, for each density gradient length and probing frequency that were studied.

In general, spectral broadening is observed with increasing turbulence level. The usual increase in central peak amplitudes and integrated spectral powers (see also Fig. 4) with increasing turbulence level is also observed. Similar results were obtained previously with simple physical optics model. ${ }^{8}$ It is known that fluctuations moving in the poloidal direction, acting as a phase grating, may result in frequency shifted sidebands at the receiver antenna. ${ }^{14}$ The amplitudes of those sidebands are expected to be equal in conventional reflectometry, at least for geometrically symmetric

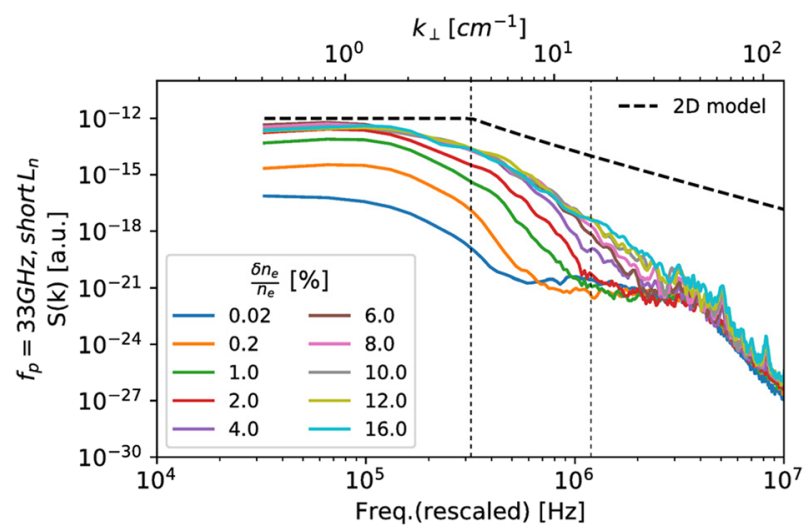

FIG. 4. Power spectra of $A(t) \mathrm{e}^{\mathrm{i} \varphi(t)}$ signals on the re-scaled single-sided frequency axis and corresponding $k_{\perp}$-axis, for a turbulence level scan with probing frequency $f_{\mathrm{p}}=33 \mathrm{GHz}$ and short density gradient scale length $L_{\mathrm{n}}$. perturbations and low propagation velocities. On the other hand, the poloidal propagation of density structures results in radial displacements of the cutoff layer as the $2 \mathrm{D}$ structures project different radial profiles in a perpendicular line of sight. Thus, any radial movements of the reflecting layer should lead to a phase modulation of the reflected signal, resulting in a symmetric broadening of the central line in the frequency spectrum. After rescaling the frequency axis, according to the ratio of velocities, assuming $v_{\text {real }}=5 \mathrm{~km} / \mathrm{s}$ and taking into account that the frequency of the density fluctuations is related to the poloidal (or equivalently in this case, perpendicular) wavenumber $v_{\mathrm{pol}} k_{\mathrm{pol}}=2 \pi f$, the wavenumber spectra can be reconstructed. This is shown in Fig. 4 for one probing frequency and a comprehensive set of turbulence levels.

The spectral shape of the input Kolmogorov-like amplitude $k$-spectrum (shown as a dashed line) is fairly well recovered. The flat amplitude region at low- $k$ followed by a strong roll-off region is observed. The values from the original model $k_{\text {knee }}=4 \mathrm{~cm}^{-1}$ and $k=15 \mathrm{~cm}^{-1}$ ( $1 \%$ of the flat top value) are also shown in Fig. 4 by vertical dashed lines. Applying an identical spectral analysis and re-scaling procedure to $\varphi(t)$ and $A(t)$ followed by linear fitting the data in the roll-off region $\left(4 \mathrm{~cm}^{-1}<k_{\perp}<15 \mathrm{~cm}^{-1}\right.$ ) allows obtaining estimates of the spectral index which are shown in Fig. 5.

At low turbulence levels, the spectral index is mostly under-estimated. However, for $\delta n_{\mathrm{e}} / n_{\mathrm{e}}>2 \%-4 \%$, the phase spectra do allow to recover the spectral index value $(-3)$ of the 2D model, suggesting that the sensitivity to high $k$ fluctuations may increase at higher turbulence levels or that simply 2D effects farther from the cutoff become increasingly more important. On the other hand, the evolution of the integrated spectral power of the $A(t) \mathrm{e}^{\mathrm{i} \varphi(t)}$ signals with the turbulence level, displayed in Fig. 6, shows a linear relation between these two quantities only up to $\delta n_{\mathrm{e}} / n_{\mathrm{e}} \approx 2 \%-4 \%$ followed by non-linear behavior at higher turbulence levels.

A similar relation is also observed between the phase fluctuations and the turbulence level, as shown in Fig. 7. In this case, and again only for low turbulence levels, the fluctuating component of the reconstructed phase signals also scales with both $k_{\mathrm{o}}$ and $\sqrt{ } L_{\mathrm{n}}$ as prescribed by Eq. (1).
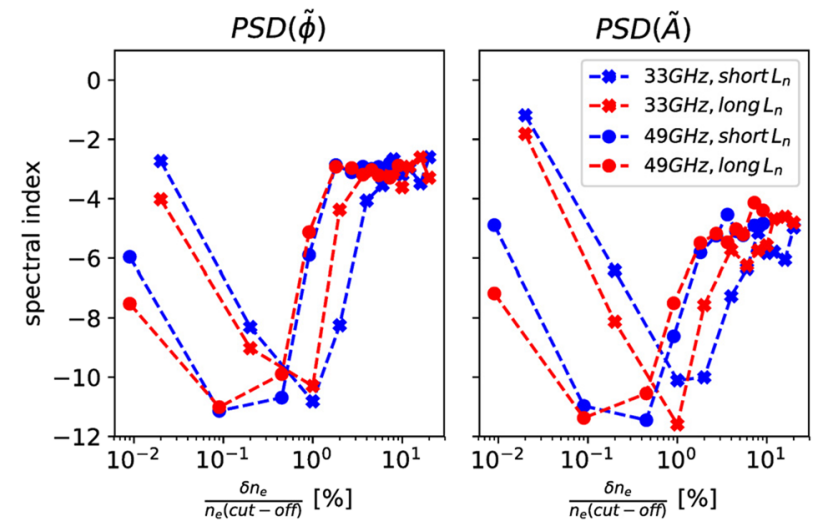

FIG. 5. Spectral index obtained from the fluctuating components of $A(t)$ and $\varphi(t)$ signals in turbulence level scans with 2 probing frequencies $f_{\mathrm{p}}$ and 2 density gradient scale lengths $L_{\mathrm{n}}$. 


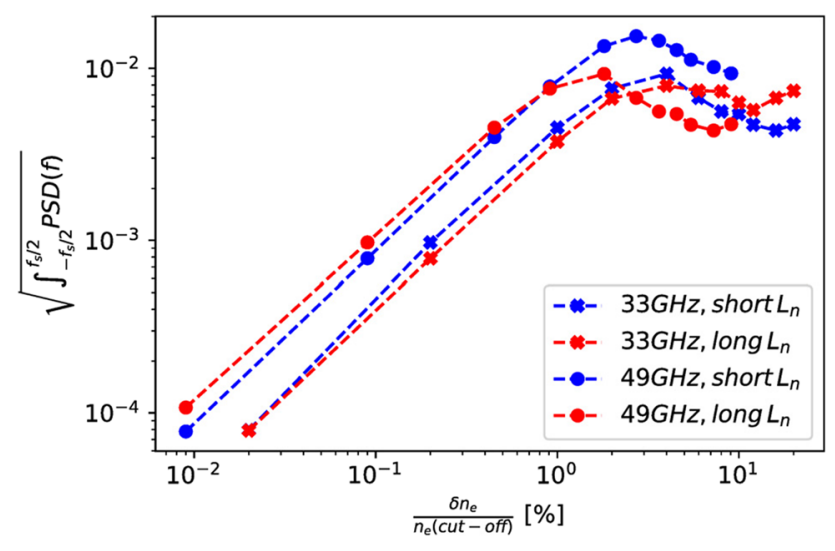

FIG. 6. Root mean square (total integrated power spectra) of $A(t) \mathrm{e}^{\mathrm{i} \varphi(t)}$ signals for turbulence level scans with 2 probing frequencies $f_{\mathrm{p}}$ and 2 density gradient scale lengths $L_{\mathrm{n}}$.

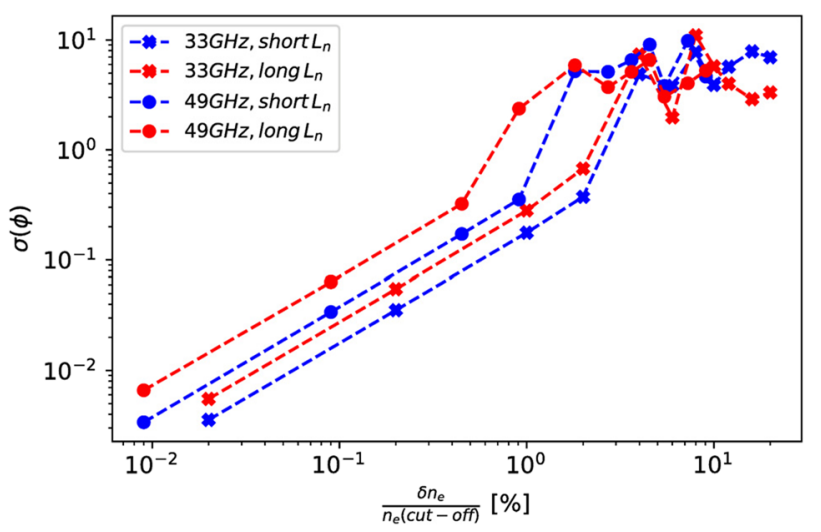

FIG. 7. Standard deviation of reflectometry $\varphi(t)$ signals for turbulence level scans with 2 probing frequencies $f_{\mathrm{p}}$ and 2 density gradient scale lengths $L_{\mathrm{n}}$.

Starting at moderate turbulence levels, the phase time traces (not shown here) display phase drifting and phase runaway effects that become ubiquitous at higher turbulence levels. The evolution of the average and fluctuating components of the reflectometry amplitude $A(t)$ is shown in Fig. 8. The average amplitude of the reflected signal decreases with increasing turbulence level and is lower, at each turbulence
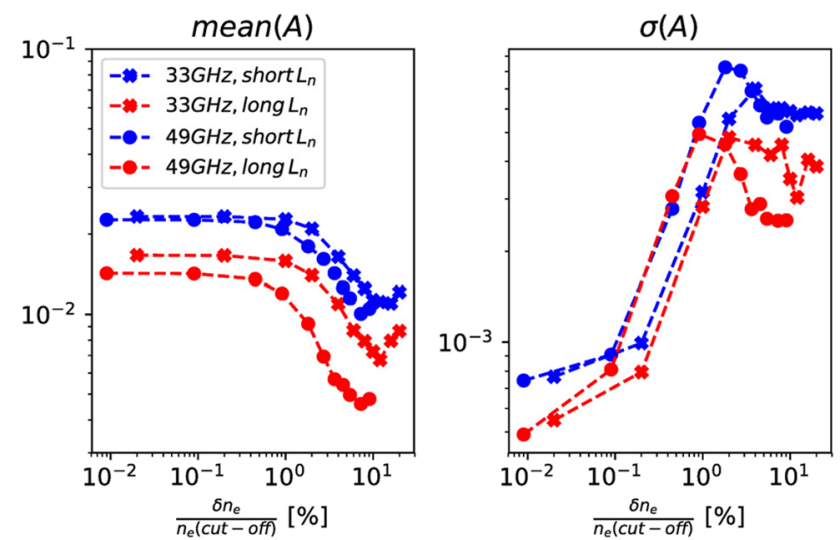

FIG. 8. Average and standard deviation of reflectometry amplitude $A(t)$ signals for turbulence level scans with 2 probing frequencies $f_{\mathrm{p}}$ and 2 density gradient scale lengths $L_{\mathrm{n}}$.

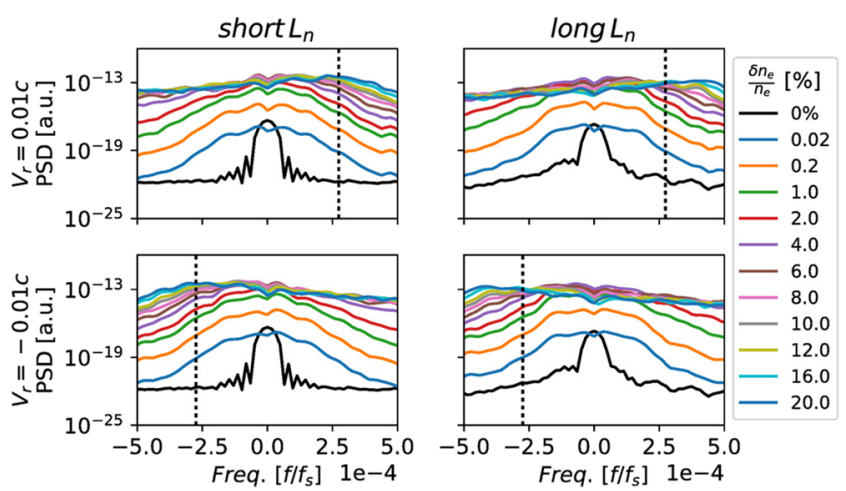

FIG. 9. Power spectra of reflectometry complex amplitude signals $A(t) \mathrm{e}^{\mathrm{i} \varphi(t)}$ for turbulence level scans in both inward and outward radial plasma flow directions, with $f_{\mathrm{p}}=33 \mathrm{GHz}$ probing frequency, and two different density gradient lengths $L_{\mathrm{n}}$ of background slab plasmas.

level, in the case of higher $L_{\mathrm{n}}$. These results must be interpreted in the light of 2D effects since 1D backscattering efficiency is proportional to $\sqrt{ } L_{\mathrm{n}}$; however, the beam spreading associated to a longer path into the turbulent plasma should provide a competing geometrical effect. ${ }^{15} \mathrm{~A}$ rebound in the average amplitude is also observed at the highest turbulence levels. This could be explained by a transition to a secondary cutoff from the slab plasma to a high amplitude density front. While the low turbulence range of linear reflectometry phase response has been previously predicted with 1D analytical reasoning, ${ }^{16} 2 \mathrm{D}$ physical optics,,${ }^{17,18}$ and full-wave models, ${ }^{18}$ these results suggest a possible extension to measuring capabilities, for instance, through proper modeling of the amplitude signals.

\section{B. Radial plasma flow}

The numerical plasmas employed in Sec. V A were also used in simulations with radial plasma flow in both the inward $\left(V_{\mathrm{r}}>0\right)$ and outward $\left(V_{\mathrm{r}}<0\right)$ directions. The double-sided Fourier spectra of the complex amplitude signals obtained with the $f_{\mathrm{p}}=33 \mathrm{GHz}$ cases are shown in Fig. 9 .

The effect of spectral broadening with increasing turbulence levels is observed again. This is more pronounced than in the poloidal flow cases, shown previously in Fig. 3. The spectra become increasingly asymmetric with increasing

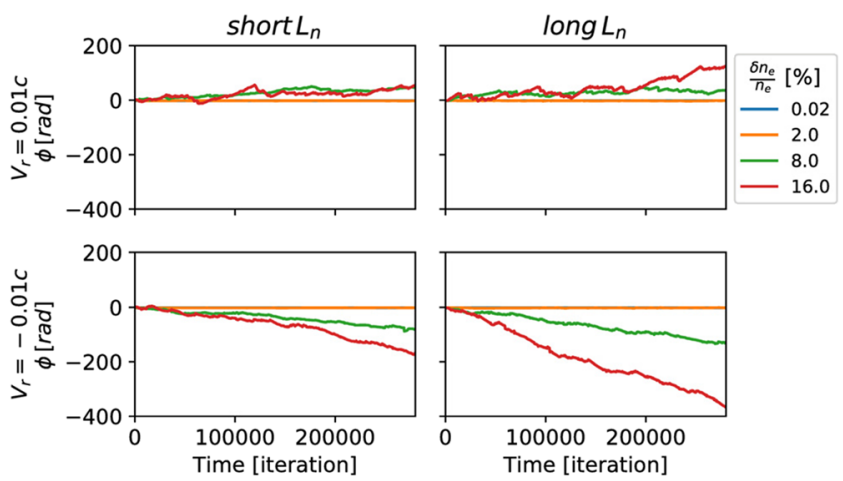

FIG. 10. Time traces of reflectometry $\varphi(t)$ signals for turbulence level scans with $f_{\mathrm{p}}=33 \mathrm{GHz}$ probing frequency and 2 density gradient scales $L_{\mathrm{n}}$ for both cases of inward and outward radial plasma flow. 


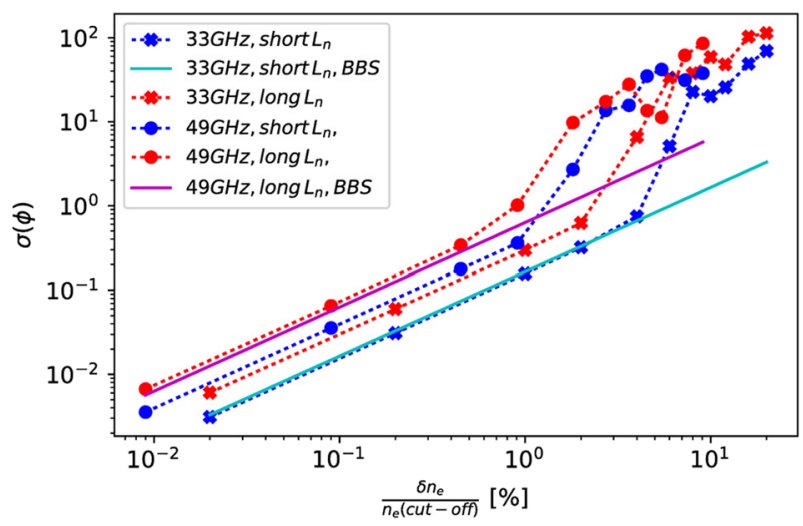

FIG. 11. Standard deviation of reflectometry $\varphi(t)$ signals for turbulence level scans with 2 probing frequencies $f_{\mathrm{p}}$ and 2 density gradient scale lengths $L_{\mathrm{n}}$, for outward radial plasma flows. Theoretical values estimated from Eq. (1) for two of the simulated cases are also shown in solid lines.

turbulence levels. The radial flow can induce Doppler effects at both the cutoff and Bragg backscattering locations. A single sideband can thus be produced by a density fluctuation with $k_{\text {rad }}=2 k(\boldsymbol{r})$ propagating radially. The maximum Doppler shifts expected by the imposed radial velocity on the cutoff layer are shown in Fig. 9 by dashed vertical lines. In cases of short $L_{\mathrm{n}}$, the observed Doppler shift is in agreement with the reflecting layer movement. For long $L_{\mathrm{n}}$, the Doppler shifts are larger due to the increased plasma path and Bragg contributions. This is additionally leading to phase runaway, as seen in Fig. 10, where the phase also changes sign with opposite propagation directions.

Despite this, phase fluctuations behave similarly for the inward and outward directions at low turbulence levels, becoming somewhat distinct at high $\delta n_{\mathrm{e}} / n_{\mathrm{e}}$ possibly due to trapped waves responding differently when being pushed out or into the probing wave. For low $\delta n_{\mathrm{e}} / n_{\mathrm{e}}$ values, phase fluctuations scale linearly as predicted by Eq. (1) and shown in Fig. 11 where an average (effective) $k_{\mathrm{f}}=3 \mathrm{~cm}^{-1}$ was assumed to calculate the theoretical Bragg Backscattering values for two of the outward propagation cases.

Standard deviation values $\sigma \varphi(t)$ largely exceeding $2 \pi$ are perhaps a good proxy for the phase drift slope rather than the phase fluctuations. Nevertheless, this quantity seems to be useful in tracking (quasi-linearly) the turbulence level. The reduction in the average amplitude with both increasing turbulence levels and density gradient length is also observed again, as displayed in Fig. 12. Nevertheless, it is arguable for both $A(t)$ and $\varphi(t)$ fluctuations (and for radial and poloidal plasma flow cases for that matter) if non-linear or saturation regimes are reached at high $\delta n_{\mathrm{e}} / n_{\mathrm{e}}$. Further simulations, extending the turbulence level range, are planned to clarify this behavior.

Also, simulations with plasmas obtained from the output of gyro-fluid or gyro-kinetic turbulence codes are ongoing
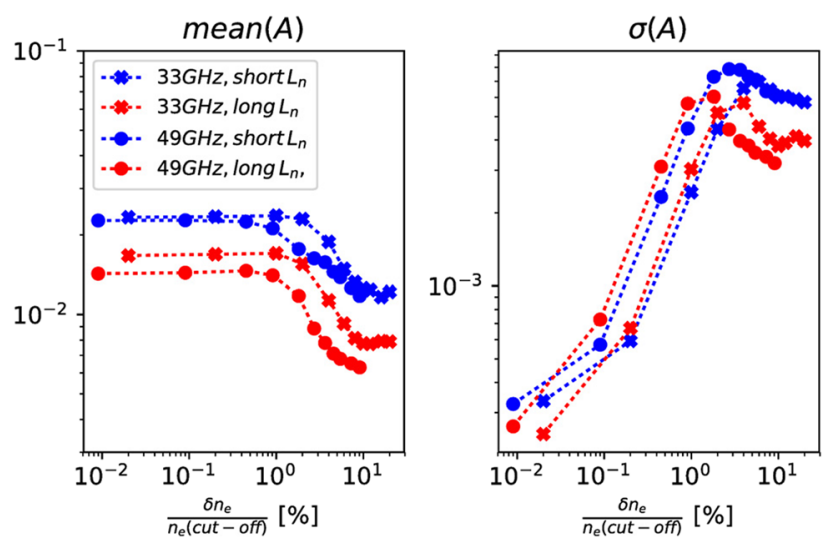

FIG. 12. Average and standard deviation of the reflectometry amplitude $A(t)$ signals for turbulence level scans in the case of outward radial plasma flow for 2 probing frequencies $f_{\mathrm{p}}$ and 2 density gradient scale lengths $L_{\mathrm{n}}$.

to have more realistic plasma models, which can then be benchmarked against the results presented here.

\section{ACKNOWLEDGMENTS}

This work has been carried out within the framework of the EUROfusion Consortium and has received funding from the Euratom research and training programme 2014-2018 under Grant Agreement No. 633053. The views and opinions expressed herein do not necessarily reflect those of the European Commission. IST activities also received financial support from "Fundação para a Ciência e Tecnologia" through Project No. UID/FIS/50010/2013.

${ }^{1}$ F. da Silva, S. Heuraux, E. Gusakov, and A. Popov, IEEE Trans. Plasma Sci. 38, 2144 (2010).

${ }^{2}$ N. Bretz, Phys. Fluids B 4, 2414 (1992).

${ }^{3}$ I. H. Hutchinson, Plasma Phys. Controlled Fusion 34, 1225 (1992).

${ }^{4}$ X. L. Zou, L. Laurent, and J. M. Rax, Plasma Phys. Controlled Fusion 33, 903 (1991).

${ }^{5}$ C. Fanack et al., Plasma Phys. Controlled Fusion 38, 1915 (1996).

${ }^{6}$ B. B. Afeyan et al., Plasma Phys. Controlled Fusion 37, 315 (1995).

${ }^{7}$ I. Boucher et al., Plasma Phys. Controlled Fusion 40, 1489 (1998).

${ }^{8}$ G. D. Conway, L. Schott, and A. Hirose, Rev. Sci. Instrum. 67, 3861 (1996).

${ }^{9}$ S. Heuraux et al., Rev. Sci. Instrum. 74, 1501 (2003).

${ }^{10}$ M. A. Pedrosa et al., Phys. Rev. Lett. 82, 3621 (1999).

${ }^{11}$ S. J. Zweben et al., Plasma Phys. Controlled Fusion 49, S1 (2007).

${ }^{12}$ G. I. Taylor, Proc. R. Soc. London, Ser. A 164, 15 (1938).

${ }^{13}$ F. da Silva, S. Heuraux, S. Hacquin, and M. E. Manso, J. Comput. Phys. 203, 467 (2005).

${ }^{14}$ E. Holzhauer, M. Hirsch, T. Grossmann, B. Brañas, and F. Serra, Plasma Phys. Controlled Fusion 40, 1869 (1998).

${ }^{15}$ E. V. Sysoeva, E. Gusakov, and S. Heuraux, Plasma Phys. Controlled Fusion 55, 115001 (2013).

${ }^{16}$ E. Gusakov, S. Heuraux, and A. Yu Popov, Plasma Phys. Controlled Fusion 51, 065018 (2009).

${ }^{17}$ G. D. Conway, Plasma Phys. Controlled Fusion 41, 65 (1999).

${ }^{18}$ J. R. Pinzón, T. Happel, E. Blanco, G. D. Conway, T. Estrada, and U. Stroth, Plasma Phys. Controlled Fusion 59, 035005 (2017). 